Artigo Original

Original Article

Letícia Caldas Teixeira ${ }^{1}$

Ana Luíza Vilar Rodrigues

Áudrea Fernanda Girundi da Silva ${ }^{2}$

Renata Azevedo ${ }^{3}$

Ana Cristina Côrtes Gama ${ }^{1}$

Mara Behlau ${ }^{4}$

Descritores

Voz

Distúrbios da voz

Reabilitação

Motivação

Conduta de saúde

Keywords

Voice

Voice disorders

Rehabilitation

Motivation

Health behavior

Endereço para correspondência:

Letícia Caldas Teixeira

R. Grão Mogol, 689, Carmo, Belo Horizonte (MG), Brasil, CEP: 30310-010.

E-mail: 1caldas4@gmail.com

Recebido em: 20/3/2011

Aceito em: 12/12/2011

\section{Escala URICA-VOZ para identificação de estágios de adesão ao tratamento de voz}

\section{The use of the URICA-VOICE questionnaire to identify the stages of adherence to voice treatment}

\section{RESUMO}

Objetivos: Adaptar a escala URICA à avaliação dos estágios de mudança para a adesão de pacientes em tratamentos de voz; e verificar a relação destes com a idade do paciente, o tipo de disfonia, a escolaridade, a profissão e o número de sessões de fonoterapia. Métodos: Estudo transversal, com amostra de conveniência, composta por 66 pacientes com disfonia, sendo 58 mulheres e oito homens, com idade entre 18 e 65 anos, atendidos em dois ambulatórios de instituições de ensino de Fonoaudiologia. Como instrumento exploratório, procedeu-se à adaptação brasileira da escala URICA para a área de voz, tendo como base situações específicas relacionadas ao comportamento vocal, denominada URICA-VOZ. O instrumento foi aplicado individualmente e as variáveis foram relacionadas. Resultados: A maioria dos pacientes, 38 (57,6\%), concentrou-se no estágio de contemplação, 20 (30,3\%) no de pré-contemplação e apenas oito $(12,1 \%)$ no de ação, ideal para a terapia fonoaudiológica. Não houve associação entre os estágios de adesão com as variáveis idade, tipo de disfonia, escolaridade e número de sessões de fonoterapia. A variável profissão apresentou associação com o estágio de ação URICA-VOZ. Conclusão: O uso da escala URICA-VOZ revelou que a maioria dos pacientes com disfonia em tratamento ainda se encontra no estágio de contemplação, o que pode restringir os resultados da terapia. Não houve relação entre as demais variáveis estudadas e os estágios de adesão do protocolo URICA-VOZ.

\begin{abstract}
Purpose: To adapt the URICA questionnaire to assess the motivational stages of patients undergoing voice treatment, and to determine the association between these stages with age, type of dysphonia, level of education, profession, and number of voice therapy sessions. Methods: A cross-sectional study design was employed, using a convenience sample composed of 66 dysphonic patients - 58 women and eight men - aged from 18 to 68 years, who were enrolled in outpatient care at two federal universities. As an exploratory tool, the URICA questionnaire was adapted to the area of voice, based on specific situations related to vocal behaviors. This questionnaire was named URICA-VOICE. It was administered individually and subjected to analyses. Results: Most patients, 38 (57.6\%), were in the contemplation stage, 20 (30.3\%) were in the pre-contemplation, and only eight $(12.1 \%)$ in the action stage, which is ideal to voice therapy. There was no association between adherence stages and the variables age, type of dysphonia, level of education, and number of voice therapy sessions. The variable profession was associated with the action stage of the URICA-VOICE. Conclusion: The URICA-VOICE showed that most dysphonic patients undergoing treatment are still in the contemplation stage, which may limit therapy outcomes. There was no relationship between the other variables and the adherence stages of the URICA-VOICE questionnaire.
\end{abstract}

Trabalho realizado no Curso de Fonoaudiologia, Universidade Federal de São Paulo - UNIFESP - São Paulo (SP) e Universidade Federal de Minas Gerais - UFMG - Belo Horizonte (MG), Brasil.

(1) Departamento de Fonoaudiologia, Faculdade de Medicina, Universidade Federal de Minas Gerais -UFMG - Belo Horizonte (MG), Brasil.

(2) Curso de Fonoaudiologia, Universidade Federal de Minas Gerais - UFMG - Belo Horizonte (MG), Brasil. (3) Departamento de Fonoaudiologia, Universidade Federal de São Paulo -UNIFESP - São Paulo (SP), Brasil (4) Programa de Pós-Graduação em Distúrbios da Comunicação Humana, Campo Fonoaudiológico, Universidade Federal de São Paulo - UNIFESP - São Paulo (SP), Brasil.

Conflito de interesses: Não 


\section{INTRODUÇÃO}

A ciência contempla estudos sobre adesão em várias áreas. Há diversos estudos e protocolos desenvolvidos para avaliar a adesão em tratamentos de saúde, como na Fisioterapia ${ }^{(1)}$, na Nutrição ${ }^{(2)}$ e na Clínica Médica ${ }^{(3)}$. Na Fonoaudiologia, todavia, especialmente nos tratamentos de voz, há carência e demanda por este tipo de estudo ${ }^{(4,5)}$, uma vez que a terapia vocal é um tratamento longitudinal, que demanda mudanças e/ou eliminação de comportamentos vocais abusivos ou nocivos para a voz, com a participação ativa do paciente.

Com base nessa vertente, torna-se importante explorar e criar ferramentas investigativas para elucidar os aspectos que permeiam a adesão na terapia de voz.

Um estudo que avaliou dois métodos de tratamento fonoterápico para pacientes com nódulos vocais reforça essa ideia ao constatar que a adesão ao tratamento tem maior impacto nos resultados do que propriamente o tipo de terapia utilizada. Os resultados mostraram que os pacientes que se consideraram aderentes à terapia apresentam maiores benefícios do que aqueles que se declararam não aderentes ${ }^{(6)}$.

A adesão, entretanto, é multifatorial e pode estar relacionada a fatores externos e internos ao indivíduo ${ }^{(7,8)}$, como idade, sexo, duração da terapia e necessidade de mudanças no estilo de vida do paciente com o tratamento ${ }^{(9,10)}$.

Para explorar os aspectos ligados à adesão, alguns modelos teóricos são utilizados, por exemplo: teoria da ação racional (Theory of Reasoned Action), modelo de crenças em saúde (Health Belief Model), teoria social cognitiva (Health applications of Social Cognitive Theory ${ }^{(5)}$ e modelo transteórico (MTT), desenvolvido por Prochaska e DiClemente, em 1982, para explicitar a autopercepção do estágio de prontidão de pacientes para tratamentos de saúde ${ }^{(11)}$. O MTT tem sido utilizado como um guia para o desenvolvimento de intervenções que implicam as mudanças de comportamento em saúde que ocorrem sob a forma de estágios, e não de maneira súbita ou num único evento ${ }^{(11)}$.

Os estágios empiricamente definidos se dividem em: pré-contemplação - o sujeito ainda não tem consciência de que há um problema a ser enfrentado; contemplação - o sujeito considera seriamente a possibilidade de enfrentar o problema, mas nenhum esforço efetivo é feito com esse objetivo; ação - as tentativas para a mudança são evidentes; e manutenção - ausência de recaídas, esforço contínuo por parte do sujeito ${ }^{(11-13)}$.

Com apoio no MTT, desenvolveu-se a escala University of Rhode Island Change Assessment (URICA), utilizada para medir as dimensões de prontidão para mudança em indivíduos que se submetem a tratamentos de saúde ${ }^{(8)}$. Estudo sugere que o modelo transteórico (MTT) de mudança de comportamento pode ser de grande utilidade na área de voz, uma vez que a maioria dos problemas de voz depende da habilidade do paciente em realizar mudanças de comportamento, além de exigir sua prontidão para iniciar esse processo. Embora este modelo não tenha sido ainda aplicado na área de voz, os autores ressaltam que ele pode ser bastante útil para a fonoterapia em $\mathrm{voz}^{(5)}$.

A possibilidade de cotejar o MTT com a terapia vocal instigou-nos a adaptar a escala URICA para a área de voz.
Não nos caberá neste estudo validar essa escala enquanto um protocolo. Esta ferramenta metodológica, denominada de URICA-VOZ, contempla a necessidade de mapear os estágios de adesão autopercebidos pelos pacientes em tratamento. Tratase de um primeiro movimento investigativo a ser usado para sinalizar ou não a sua utilidade na área da voz.

Esta pesquisa teve por objetivo adaptar a escala URICA para avaliar os estágios de prontidão de pacientes para a adesão em tratamento de voz, contemplando as variáveis idade do paciente, tipo de disfonia, escolaridade, profissão e número de sessões de fonoterapia.

\section{MÉTODOS}

Para participar desta pesquisa, foram convidados todos os pacientes $(\mathrm{n}=80)$ que estavam em fonoterapia nos ambulatórios de voz dos Hospitais Universitários de duas instituições de ensino em Fonoaudiologia: Ambulatório de Fonoaudiologia do Hospital das Clínicas da Universidade Federal de Minas Gerais e Ambulatório de Voz do Hospital São Paulo, da Universidade Federal de São Paulo, no período de março a junho de 2010.

A amostra foi composta por 66 pacientes, uma vez que 14 pacientes (20\%) não aceitaram participar do estudo, configurando um erro amostral de 5\% e nível de confiança de $95 \%$. Os participantes situaram-se em 18 e 68 anos de idade (média $=42,27 ;$ mediana $=41,00)$. Quanto ao gênero, $58(87,9 \%)$ eram mulheres, enquanto $8(12,1 \%)$ eram homens. A escolaridade variou, sendo composta em sua maior parte por graduados $52(78,8 \%)$. Quanto à profissão, 48 (72,7\%) eram profissionais da voz 48 (72,7\%). O número de sessões de fonoterapia realizadas variou de 1 a 49 (média=12,06; mediana=10,0). Apuramos também que 60 pacientes $(90,9 \%)$ apresentavam disfonia do tipo comportamental e $6(9,1 \%)$, disfonia não comportamental.

Como critérios de inclusão, consideraram-se todos os pacientes com diagnóstico clínico de disfonia que estavam iniciando tratamento fonoaudiológico há, pelo menos, uma semana, após avaliação inicial. Nenhum dos participantes desta pesquisa estava sendo atendido por qualquer dos autores deste estudo. Portanto, eles não tinham contato direto com as pesquisadoras. Igualmente, nenhum paciente teve dificuldade em compreender as questões formuladas.

Esta pesquisa constou de dois momentos: 1) análise dos prontuários, para determinar as variáveis sexo, idade, tipo de disfonia, escolaridade, profissão e número de sessões de fonoterapia; e 2) aplicação da escala, para o mapeamento da autopercepção do estágio de adesão dos pacientes disfônicos à terapia de voz.

A variável escolaridade foi dividida em: graduados - ensino inferior incompleto ou completo; e não graduados - ensino fundamental ou médio incompleto ou completo. A variável profissão incluiu como profissionais da voz os sujeitos que usam a voz como ferramenta de trabalho ${ }^{(14,15)}$, e não os profissionais da voz, ou seja, aqueles que não dependem desta para exercer a profissão. A variável tipo de disfonia foi classificada em: comportamental - baseada no comportamento vocal como resultado; e não comportamental - baseada numa alteração estrutural independente do comportamento vocal. 
A escala URICA, amplamente utilizada em pesquisas para avaliar a autopercepção do estágio de adesão em tratamentos de saúde ${ }^{(11,12,16)}$, foi adaptada por cinco especialistas em voz para um modelo de autopercepçao da adesão na área de voz, considerando-se aspectos do comportamento e do uso vocal.

A escala URICA original apresenta versão de 12, 24 e 32 itens. A versão utilizada para a adaptação na área da voz foi a de 32 itens, assim eleita por ser a mais completa e por ser a primeira vez que a escala seria adaptada para a área de voz. Nesta versão, os itens são divididos em quatro grupos de oito afirmações, correspondentes aos quatro estágios de mudança: pré-contemplação; contemplação; ação; e manutenção(13). As perguntas referentes a cada um desses estágios são: pré-contemplação: 1, 5, 11, 13, 23, 26, 29 e 31; contemplação: 2, $4,8,12,15,19,21$ e 24 ; ação: $3,7,10,14,17,20,25$ e 30 ; e manutenção: $6,9,16,18,22,27,28$ e 32. Para cada item são apresentadas possibilidades de respostas em escala do tipo likert de cinco pontos, na qual o sujeito pode escolher uma das seguintes respostas: "Discordo totalmente", "Discordo", "Não sei", "Concordo" e "Concordo totalmente".

Mantivemos o nome URICA e acrescentamos ao título a palavra VOZ, uma vez que sua construção se baseou no modelo da escala URICA e seu objetivo consistiu em verificar aspectos relacionados à voz (Anexo 1). A adaptação foi feita com o conhecimento e a permissão dos criadores do instrumento original.

Duas fonoaudiólogas, pesquisadoras deste estudo e fluentes da língua inglesa traduziram, de modo independente, a escala original para o Português. Depois de discutida as traduções, uma única versão foi considerada e procedeu-se à contraversão e à comparação com a versão original do inglês, acrescida dos aspectos relativos à voz. A versão em Português foi analisada quanto aos aspectos de facilidade de compreensão por uma terceira fonoaudióloga, fazendo-se os ajustes necessários.

Mantivemos a referência conceitual da afirmação, certificando-nos de que sua adaptação não comprometeria a correspondência com o estágio de mudança ao qual originalmente se referia. Dessa forma, por exemplo, a questão 1, que na escala original era "I don't have any problems that need changing" (correspondente ao estágio de adesão de pré-contemplação), foi traduzida e adaptada na URICA-VOZ como "Acredito que eu não tenho que mudar minha voz". A questão 30, que na URICA é "I am actively working in my problem" (ação), foi traduzida na URICA-VOZ como: "Eu estou trabalhando ativamente para resolver meu problema de voz".

Em seguida, uma versão piloto foi aplicada por duas autoras deste estudo em dez pacientes com problemas de voz, com tipos diferentes de disfonia, para validação do conteúdo das afirmações. Ao lado de cada uma das sentenças do protocolo havia a possibilidade de responder "Não se aplica" caso o paciente considerasse a afirmação inadequada em relação ao seu problema de voz. Nenhum paciente assinalou essa possibilidade. Assim, todas as afirmações foram consideradas aplicáveis e nenhuma questão precisou ser refeita ou eliminada. Em média, os pacientes gastaram de três a cinco minutos para responder ao questionário.

Em relação ao cálculo da URICA, usamos a pontuação da escala proposta pela Healthy and Addictive Behaviors:
Investigating Transtheorical Solutions (HABITS), da University of Maryland, Baltimore County ${ }^{(13)}$. Tal opção de análise já foi usada em outras publicações ${ }^{(12,13)}$, inclusive na adaptação brasileira da escala para a análise de usuários de drogas ilícitas ${ }^{(12)}$. Conforme orientação da HABITS, deve-se, inicialmente, efetuar uma média simples das marcações das afirmações correspondentes a cada estágio de mudança, excluindo-se as questões 4, 9, 20, 1 e 31, que não entram nos cálculos.

O escore de prontidão para a mudança prevê o resultado de um tratamento, no início deste, identificando se o indivíduo está ou não em uma fase adequada do ciclo de mudança para ser submetido à intervenção proposta. Dessa forma, cada estágio fica representado pela média de sete questões. Para obter-se o escore da prontidão para a mudança, aplica-se a seguinte fórmula: (Média de C + Média de A + Média de M) - Média de PC.

Ou seja, somatória da média dos resultados dos estágios de contemplação $(C)$, ação $(A)$ e manutenção $(M)$, seguida de subtração do resultado do valor médio do estágio de pré-contemplação (PC). As pontuações dos cortes são:

- 8 ou inferior - para os sujeitos em fase de pré-contemplação;

- 8-11 - para os sujeitos em fase de contemplação; e

- 11-14 - para os sujeitos que estão preparados para uma atitude de ação diante do problema ${ }^{(13)}$.

A análise estatística das variáveis estudadas foi realizada por meio das medidas de tendência central e de dispersão e de testes de hipóteses em nível de significância de 5\%. Para as variáveis categóricas, foi aplicado o teste Qui-quadrado e para as variáveis contínuas, a análise ANOVA, por meio do programa estatístico SPSS 170.

Este estudo foi aprovado pelos Comitês de Ética da Universidade Federal de São Paulo (UNIFESP) e da Universidade Federal de Minas Gerais (UFMG) (0283/10 e ETIC 603/09). Todos os sujeitos que concordaram em participar da pesquisa assinaram o Termo de Consentimento Livre e Esclarecido (TCLE).

\section{RESULTADOS}

Em relação aos estágios do protocolo URICA-VOZ (Tabela 1), 38 pacientes $(57,6 \%)$ concentraram-se no estágio de contemplação; 20 (30,3\%) no estágio de pré-contemplação; e oito $(12,1 \%)$, no estágio de ação. Nenhum dos pacientes estava no estágio de manutenção.

No estágio de pré-contemplação, 16 sujeitos $(80,0 \%)$ eram graduados e quatro $(20,0 \%)$ não graduados. No estágio

Tabela 1. Autopercepção da adesão em tratamento de voz

\begin{tabular}{lcc}
\hline Estágio & $\mathrm{n}$ & $\%$ \\
\hline $\mathrm{PC}$ & 20 & 30,3 \\
$\mathrm{C}$ & 38 & 57,6 \\
$\mathrm{~A}$ & 8 & 12,1 \\
$\mathrm{M}$ & 0 & 0 \\
\hline Total & 66 & 100 \\
\hline
\end{tabular}

Legenda: $P C=$ pré-contemplação; $C$ = contemplação; $A=$ ação; $M=$ manutenção 
Tabela 2. Associação da URICA-VOZ com as variáveis categóricas

\begin{tabular}{|c|c|c|c|c|}
\hline Variáveis categóricas & $\begin{array}{c}\mathrm{PC} \\
\mathrm{n}(\%)\end{array}$ & $\begin{array}{c}\mathrm{C} \\
\mathrm{n}(\%) \\
\end{array}$ & $\begin{array}{c}\text { A } \\
\mathrm{n}(\%) \\
\end{array}$ & Valor de $p$ \\
\hline \multicolumn{5}{|l|}{ Escolaridade } \\
\hline Graduados & $16(80)$ & $32(84,2)$ & $4(50)$ & 0,098 \\
\hline Não-graduados & $4(20)$ & $6(15,8)$ & $4(50)$ & \\
\hline \multicolumn{5}{|l|}{ Profissão } \\
\hline Profissionais & $17(85)$ & $30(78,9)$ & $1(12,5)$ & $0,001^{*}$ \\
\hline \multicolumn{5}{|l|}{ Diagnóstico fonoaudiológico } \\
\hline Comportamental & $20(100)$ & $34(89,5)$ & $6(75)$ & 0,224 \\
\hline Não-comportamental & $0(0)$ & $4(10,5)$ & $2(25)$ & \\
\hline
\end{tabular}

*Valores significativos $(p<0,05)-$ Teste Qui-quadrado

Legenda: $\mathrm{PC}=$ pré-contemplação; $\mathrm{C}=$ contemplação; $\mathrm{A}=$ ação

de contemplação, 32 sujeitos $(84,2 \%)$ eram graduados e seis $(15,8 \%)$ não graduados. No estágio de ação, quatro sujeitos $(50,0 \%)$ eram graduados e quatro $(50 \%)$ não graduados (Tabela 2). Não houve diferença entre a variável escolaridade e os estágios de adesão do protocolo.

No estágio de pré-contemplação, 17 sujeitos (85\%) eram profissionais da voz e três $(15,0 \%)$ não eram profissionais da voz. No estágio de contemplação, 30 (78,9\%) sujeitos eram e oito $(21,1 \%)$ não eram profissionais da voz. No estágio de ação, um paciente $(12,5 \%)$ era e sete $(87,5 \%)$ não eram profissionais da voz (Tabela 2). A variável profissão apresentou associação com o estágio de ação URICA-VOZ ( $<<0,001)$. Nos estágios de pré-contemplação e de contemplação, a maioria dos pacientes era composta de profissionais da voz. No estágio de ação, apenas um paciente era profissional da voz.

No estágio de pré-contemplação, todos os 20 sujeitos $(100,0 \%)$ apresentavam disfonia comportamental e nenhum apresentava disfonia não comportamental. No estágio de contemplação, 34 sujeitos $(89,5 \%)$ apresentavam disfonia comportamental e quatro (10,5\%) disfonia não comportamental. No estágio de ação seis sujeitos $(75,0 \%)$ apresentavam disfonia comportamental e dois $(25,0 \%)$ disfonia não comportamental (Tabela 2). Não houve diferença entre a variável tipo de disfonia e os estágios de adesão do protocolo URICA-VOZ ( $\mathrm{p}=0,224)$.

No estágio de pré-contemplação, a média de sessões foi de 10,10. No estágio de contemplação, a média foi de 12,89 . No estágio de ação, a média foi de 13,00 (Tabela 3). Não houve diferença entre a variável número de sessões de fonoterapia e os estágios de adesão do protocolo URICA-VOZ $(\mathrm{p}=0,058)$.

No estágio de pré-contemplação, a média de idade foi de 42,70 anos (DP=11,992). No estágio de contemplação, a média de idade foi de 41,97 anos ( $\mathrm{DP}=10,729)$. No estágio de ação, a média foi de 42,63 anos (DP=16,008) (Tabela 3). Não houve diferença entre a variável idade e os estágios de adesão do protocolo URICA-VOZ ( $\mathrm{p}=0,972)$.

\section{DISCUSSÃO}

Grande parte dos pacientes desta pesquisa encontra-se nos estágios de contemplação e de pré-contemplação (Tabela 1), nos quais ainda assumiram uma atitude de enfrentamento diante do
Tabela 3. Associação da URICA-VOZ com as variáveis contínuas

\begin{tabular}{lcccc}
\hline Estágio & $\mathrm{n}$ & $\mathrm{M}$ & $\mathrm{DP}$ & Valor de $\mathrm{p}$ \\
\hline Número de sessões & & & & \\
PC & 20 & 10,10 & 7,840 & \\
C & 38 & 12,89 & 10,790 & 0,0580 \\
A & 8 & 13,00 & 11,071 & \\
Total & 66 & 12,06 & 9,957 & \\
\hline Idade & & & & \\
PC & 20 & 42,70 & 11,992 & \\
C & 38 & 41,97 & 10,729 & 0,972 \\
A & 8 & 42,63 & 16,008 & \\
Total & 66 & 42,27 & 11,631 & \\
\hline
\end{tabular}

ANOVA $(p<0,05)$

Legenda: $P C=$ pré-contemplação; $C=$ contemplação; $A=$ ação; $M=$ média; $\mathrm{DP}=$ desvio-padrão

problema. Portanto, não reúnem as melhores condições para mudar de comportamento vocal. A adesão de pacientes à terapia de voz é um desafio para a maioria dos fonoaudiólogos, pois prognósticos satisfatórios dependem da ação do paciente em relação ao tratamento. Dessa forma, um trabalho prévio deve ser desenvolvido para melhorar as chances de adesão e alcançar resultados futuros mais positivos. Autores reconhecem que entre os pacientes que se submetem a tratamentos de voz existem dois tipos de comportamento diferentes: não revelar consciência da possibilidade de mudança no comportamento ou dos benefícios dessa mudança; e revelar consciência, mas optar por não realizar mudanças, porque estão satisfeitos em saber que a causa da disfonia não é uma doença grave, como um câncer(5).

Apenas oito sujeitos desta pesquisa se perceberam no estágio de ação, em que o paciente atua no sentido de fazer alguma coisa para mudar os comportamentos relacionados à voz. Eles usam as sessões para rever exercícios vocais e discutir com o terapeuta as dificuldades que encontram para utilizar novos comportamentos benéficos para a voz ${ }^{(5)}$. Os dados reforçam que a maioria dos pacientes não iniciou verdadeiramente uma ação para mudar de comportamento.

Nenhum dos sujeitos se reconheceu no estágio de manutenção, caracterizado pela existência de todos os esforços 
para impedir o retorno aos padrões anteriores e consolidar os ganhos $^{(12)}$. Aqui, os pacientes deveriam conseguir manter sua voz saudável e os comportamentos favoráveis à saúde vocal de forma habitual. Ser capaz de controlar um episódio de disfonia e de restaurar a voz, nessa situação, são atitudes que fazem parte do sucesso deste estágio ${ }^{(5)}$.

Quanto à variável escolaridade, não encontramos relação significante com os estágios da adesão, contrariando a literatura. Um estudo mostrou que os sujeitos com maior escolaridade em tratamento antirretroviral foram mais aderentes ao tratamento do que os não escolarizados ${ }^{(17)}$. Uma pesquisa que investigou a influência do letramento com a adesão a tratamentos de saúde encontrou que o baixo letramento em saúde está associado a maior dificuldade de adesão ao tratamento medicamentoso ${ }^{(18)}$.

Alguns autores que pesquisaram a adesão em tratamentos de saúde ainda afirmam que quanto mais baixo o nível de escolaridade maior a possibilidade de não adesão ao tratamento por parte do paciente ${ }^{(19,20)}$. Uma pesquisa mostrou que grau de escolaridade mais alto dos pais e/ou responsáveis de crianças em tratamento fonoaudiológico influencia positivamente o esclarecimento da terapia fonoaudiológica realizada com seus filhos. Isso evidencia que a escolaridade pode ter correlação com a adesão ao tratamento, na medida em que baixa instrução pode dificultar a compreensão daquilo que é proposto e, consequentemente, desmotivar o indivíduo para a realização do tratamento ${ }^{(21)}$. Não foi encontrado nenhum estudo correlacionando escolaridade e adesão ao tratamento de voz. Portanto, tal aspecto deve ser investigado em futuras pesquisas.

Avaliamos a relação significativa entre a variável profissão e os estágios de adesão da escala URICA-VOZ com prudência (Tabela 2), uma vez que a grande porcentagem de pacientes profissionais da voz em nosso estudo pode ter influenciado os resultados. Pesquisas futuras que controlem este aspecto na amostra tornam-se importantes para o entendimento mais robusto desta variável na adesão/não adesão em tratamentos de voz. Entretanto, deve-se reforçar que a variável profissão deve ser analisada com atenção nas terapias de voz, uma vez que os profissionais da voz dependem da voz como ferramenta de trabalho ${ }^{(14,15)}$. Há dados na literatura que apontam que as exigências de mudanças de comportamento são mais evidentes entre os profissionais da voz, pois eles podem apresentar uma relação diferente com a voz quando comparados aos não profissionais quanto aos aspectos de qualidade de vida. Para estes profissionais, um pequeno problema vocal pode produzir um grande impacto em sua vida ${ }^{(22,23)} \mathrm{e}$, portanto, os estágios de prontidão para adesão podem ser diferentes nesta categoria profissional.

Quanto á variável disfonia, não houve diferença entre ela e os estágios de adesão do protocolo URICA-VOZ (Tabela 2). A literatura aponta que a mudança de comportamento preconizada para os casos de disfonias comportamentais pode comprometer a eficácia da terapia de voz, pois o resultado fica na dependência do quanto e do como os pacientes aderem às recomendações do tratamento, e não necessariamente do método empregado ${ }^{(14)}$. Outro aspecto a ser mencionado é que os serviços públicos ambulatoriais têm escassez de recursos materiais, sendo que as gravações de voz são, muitas vezes, realizadas somente no momento da avaliação ou da alta do paciente, o que prejudica a percepção de melhora pelo paciente durante o processo de terapia. A motivação fica limitada pela própria falta de estímulos, que seria, em condições ideais, um recurso valioso para incentivar o paciente. Em estudo sobre fatores de interferência na adesão à terapia vocal, apurou-se que ocorre melhor adesão em pacientes que percebem a efetividade do tratamento de voz. Contudo, os próprios pacientes desta pesquisa relataram que a terapia de voz é um processo que exige empenho, uma atitude autorregulatória e uma boa aliança entre terapeuta e paciente ${ }^{(4)}$.

Pesquisa sobre dois métodos de tratamento para nódulos vocais constatou que a adesão ao tratamento tem maior impacto nos resultados do que propriamente o tipo de terapia utilizada ${ }^{(6)}$. Esses dados sugerem que o tratamento de voz exige um esforço do paciente para mudar os comportamentos e para manter novos padrões vocais adquiridos com a terapia, o que pode ser um fator de dificuldade de adesão ao tratamento vocal muito mais que o tipo de disfonia apresentada.

Os dados da Tabela 3 mostram que não houve uma relação entre a variável número de sessões de fonoterapia e os estágios de adesão. De acordo com a literatura, o tempo de terapia no caso de disfonias entre homens e mulheres varia, em média, de quatro meses e meio a dez meses ${ }^{(24)}$. Nas clínicas-escola, o atendimento de voz varia, em média, seis meses, com 15 sessões aproximadamente. No ambulatório de Fonoaudiologia do Hospital das Clínicas da UFMG, a duração do tratamento vocal é de seis meses, com média de dezenove sessões realizadas ${ }^{(25)}$.

Nessa perspectiva de tempo, quanto à alta fonoaudiológica, não fica claro, se os pacientes do nosso estudo estariam se direcionando para uma futura mudança no estágio de adesão ou se eles estariam caminhando para o término do tratamento sem modificar a atitude para a ação em relação ao tratamento. Sugere-se que em futuras pesquisas o instrumento URICAVOZ seja utilizado acompanhando-se longitudinalmente os pacientes, com a aplicação da escala no princípio, no meio e no final da terapia, com o intuito de traçar os estágios de mudança de comportamento do paciente. Os autores da escala URICA apontam que no decurso de um tratamento devem-se usar as subescalas de ação e manutenção para investigar o envolvimento do indivíduo, e não o escore completo de prontidão para mudança, pois nessa situação sua utilização já não é apropriada. As subescalas de ação e manutenção indicam atitudes e atividades relacionadas aos estágios de mudança, e não ao estado (status) do ciclo de mudança. As mudanças nos subescores da escala estão associadas ao progresso no processo de tratamento e não são de natureza linear. A manutenção é medida diretamente pelas questões que compõem essa subescala ${ }^{(13)}$.

Observando a variável idade, não foi encontrada diferença estatística com os estágios da adesão do modelo proposto para o estudo (Tabela 3). Na literatura, os dados sobre a relação entre idade e adesão são controversos. Em adesão de pacientes diabéticos à terapia medicamentosa, encontrou-se que a adesão é maior em indivíduos idosos ${ }^{(26)}$. Em estudo que analisou a adesão em terapia antirretroviral, foi encontrado que a adesão é mais satisfatória nos indivíduos mais novos ${ }^{(17)}$. No entanto, a adesão de idosos a tratamentos de saúde mostrou que a idade não tem sido consistentemente associada à adesão $0^{(27)}$. 
Um estudo mostrou que não há relação entre idade e adesão ao tratamento vocal ${ }^{(28)}$. Há contradições na literatura acerca da relação entre idade e adesão. O que se discute é que crianças e idosos apresentam maiores dificuldades de aderirem ao tratamento por dependerem de outras pessoas para, por exemplo, comparecer ao local do tratamento ${ }^{(27)}$. Do adulto se espera uma independência maior para ir e vir, não sendo este um fator a mais na gama de aspectos que permeiam a questão da adesão a um tratamento.

Algumas pesquisas já apontam a importância da motivação para a adesão ao tratamento de saúde. Em fatores associados à adesão ao tratamento farmacológico da diabetes mellitus, foi encontrado que a motivação apresenta correlação positiva com a adesão ao tratamento ${ }^{(29)}$. Na adesão de adolescentes em tratamento da dependência química, ressalta-se muito a importância da motivação no resultado satisfatório do tratamento ${ }^{(30)}$. Por isso, sugerimos que outros estudos sejam realizados nesta vertente.

Nossa experiência na aplicação da escala durante a pesquisa mostrou que a escala URICA-VOZé simples de ser aplicada. Os participantes do estudo responderam facilmente às perguntas. Embora não tenha sido feito nenhum estudo específico sobre a administração da escala, o tempo gasto para o procedimento foi inferior a cinco minutos. Sugerimos que ela seja utilizada algumas vezes ao longo da terapia, para traçar os estágios de mudança de comportamento do paciente, uma vez que o acesso do terapeuta aos resultados da URICA-VOZ durante o tratamento possibilita ao clínico trabalhar a conscientização e a motivação do paciente para tratamento vocal.

Este estudo traz limitações, e uma amostra mais representativa poderá proporcionar dados adicionais à compreensão dos aspectos relacionados à adesão para a terapia de voz. Novas pesquisas poderão identificar outros fatores que podem estar relacionados com a adesão à terapia de voz, tais como: categoria diagnóstica do distúrbio vocal, grau da disfonia, percepção da intensidade da doença, relação terapeuta-paciente, tipo de abordagem de tratamento e, principalmente, aqueles mais subjetivos, como motivação e prontidão para a mudança comportamental. Esperamos também que outros estudos avancem e que se verifique a possibilidade de validação da escala URICA-VOZ.

\section{CONCLUSÃO}

O uso da escala URICA-VOZ revelou que a maioria dos pacientes com disfonia em tratamento ainda se encontra no estágio de contemplação, o que pode restringir os resultados da terapia. Não se constatou relação entre as variáveis estudadas e os estágios de adesão do protocolo URICA-VOZ.

\footnotetext{
* Todos os autores desta pesquisa (ALVR, AFGS, ACCG, RA, LCT e $M B)$ ajudaram a construir e desenvolver o trabalho, incluindo a revisão de literatura, análise dos resultados, discussão e conclusões. RA e LCT realizaram a coleta de dados; ALVR e AFGS realizaram a coleta e a tabulação dos dados. LCT e MB realizaram a revisão final do artigo.
}

\section{REFERÊNCIAS}

1. Guimarães CQ, Teixeira-Salmela LF, Rocha IC, Bicalho LI, Sabino GS. Fatores associados à adesão ao uso de palmilhas biomecânicas. Rev Bras Fisioter. 2006 Jul-Set;10(3):271-77.

2. Nalin T, Perry IDS, Refosco LF, Oliveira Netto CB, Souza CFM, Vieira TA, et al. Fenilcetonúria no Sistema Único de Saúde: avaliação de adesão ao tratamento em um centro de atendimento no Rio Grande do Sul. Rev HCPA. 2010;30(3):225-32

3. Lunelli RP, Portal VL, Esmério FG, Moraes MA, Souza EN. Patients' with coronary disease' adherence to pharmacological and nonpharmacological therapy. ACTA Paul Enferm. 2009;22(4):367-73

4. Van Leer EV, Connor NP. Patient perceptions of voice therapy adherence. J Voice. 2010 Jul;24(4):458-69.

5. Van Leer EV, Hapner ER, Connor NP. Transtheoretical model of health behavior change applied to voice therapy. J Voice. 2008 Nov;22(6):68898.

6. Verdolini-Marston K, Burke MK, Lessac A, Glaze L, Caldwell E. Preliminary study of two methods of treatment for laryngeal nodules. J Voice. 1995 Mar;9(1):74-85.

7. Dodd KJ, Taylor NF, Denisenko S, Prasad D. A qualitative analysis of a progressive resistance exercise programme for people with multiple sclerosis. Disabil Rehabil. 2006 Sep 30;28(18):1127-34.

8. McConnaughy EA, Prochaska JO, Velicer WF. Stages of change in psychotherapy: measurement and sample profiles. Psychotherapy: Theory, Research and Pratice. 1983;20(3):368-375.

9. Berhman A, Rutledge J, Hembree A, Sheridan S. Vocal hygiene education, voice production therapy, and the role of patient adherence: a treatment effectiveness study in women with phonotrauma. J Speech Lang and Hear Res. 2008 Apr;51(2):350-66.

10. Silveira LMC, Ribeiro VMB. Grupo de adesão ao tratamento: espaço de "ensinagem" para profissionais de saúde e pacientes. Interface Comunic, Saúde, Educ. 2004 Set/2005 Fev;9(16):91-104.

11. DiClement CC, Schlundt BSD, Gemmell L. Readiness and stages of change in addiction treatment. Am J Addict. 2004 Mar-Apr;13(2):103-19.

12. Szupszynski KPDR, Oliveira MS. Adaptação brasileira da University of Rhode Island Change Assessment (URICA) para usuários de substâncias ilícitas. Psico-USF. 2008 Jun;13(1):31-9.

13. The HABITS Lab at UMBC. University of Rhode Island Change Assessment Scale: URICA. [Internet]. [cited 2011 may 11]. Available from: http://www.umbc.edu/psyc/habits/content/ttm_measures/urica/ index.html

14. Araújo TM, Reis EJFB, Carvalho FM, Porto LA, Reis IC, Andrade JM. Fatores associados a alterações vocais em professoras. Cad Saúde Pública. 2008 Jun;24(6):1229-38.

15. Behlau M, Feijó D, Madazio G, Rehder MI, Azevedo R, Ferreira AE. Voz profissional: aspectos gerais e atuação fonoaudiológica. In: Behlau M (Org.). Voz: O livro do especialista. Rio de Janeiro: Revinter; 2005. vol 2. p. 287.

16. Szupszynski KPDR, Oliveira MS. O Modelo Transteórico no tratamento da dependência química. Psicol Teor Prat. 2008 Jun;10(1):162-73.

17. Colombrini MRC, Dela Coleta MF, Lopes MHBM. Fatores de risco para a não adesão ao tratamento com terapia antiretroviral altamente eficaz. Rev Esc Enferm USP. 2008 Set;42(3):490-5.

18. Maragno CAD. Associação entre letramento em saúde e adesão ao tratamento medicamentoso [dissertação]. Rio Grande do Sul: Faculdade de Farmácia da Universidade Federal do Rio Grande do Sul; 2009.

19. Bagattoli RM, Vaisman M, Lima JS, Ward LS. Estudo de adesão ao tratamento do hipotiroidismo. Arq Bras Endocrinol Metab. 2000 Dez;44(6):483-7.

20. Bonolo PF, Ceccato MGB, Acurcio FA, Rocha GM, Guimarães MDC. Compreensão de informações relativas ao tratamento anti-retroviral entre indivíduos infectados pelo HIV. Cad Saúde Pública. 2004 Out;20(5):1388-97.

21. Nascimento IT, Teixeira LC, Zarzar PMPA. Bioética: esclarecimento e Fonoaudiologia. Rev CEFAC. 2009 Mar;11(1):158-65.

22. Murry T, Zschommler A, Prokop J. Voice handicap in singers. J Voice. 2009 May;23(3):376-9. 
23. Rosen CA, Murry T, Zinn A, Zullo T, Sonbolian M. Voice handicap index change following treatment of voice disorders. J Voice. 2000 Dec;14(4):619-23.

24. Behlau M, Madazio G, Feijó D, Azevedo R, Gielow I, Rehder MI. Aperfeiçoamento vocal e tratamento fonoaudiológico das disfonias. In: Behlau M (Org). Voz: O livro do especialista. Rio de Janeiro: Revinter; 2005. vol 2. p.409-37.

25. Bassi IB, Assunção AA, Medeiros AM, Menezes LN, Teixeira LC, Gama ACC. Quality of life, self-perceived dysphonia and diagnosed dysphonia through clinical tests in teachers. J Voice. 2011 Mar;25(2):192-201.

26. Gimenes HT, Zanetti ML, Haas VJ. Fatores relacionados à adesão do paciente diabético à terapêutica medicamentosa. Rev Lat Am Enfermagem. 2009 Jan-Fev;17(1):46-51.
27. Almeida HO, Versiani ER, Dias AR, Novaes MRCG, Trindade LMV. Adesão a tratamentos entre idosos. Comun ciên saúde. 2007 JanMar;18(1):57-67.

28. Hapner E, Portone-Maira C, Johns III MM. A study of voice therapy dropout. J Voice. 2009 May;23(3):337-40.

29. Assunção TS, Ursine PGS. Estudo de fatores associados à adesão ao tratamento não farmacológico em portadores de diabete mellitus assistidos pelo Programa Saúde da Família, Ventosa, Belo Horizonte. Cien Saude Colet. 2008 Dez;13(2):2189-97.

30. Scaduto AA, Barbieri V. O discurso sobre a adesão de adolescentes ao tratamento de dependência química em uma instituição de saúde pública. Ciênc Saúde Colet. 2009 Abr;14(2):605-14.

\section{Anexo 1. Escala URICA-VOZ*}

Queremos conhecer sua opinião sobre como você lida com sua voz. Abaixo estão escritas 32 frases que as pessoas usam sobre isso. Por favor, leia com atenção e marque o quanto você discorda ou concorda com as afirmações. Não existe certo ou errado, elas apenas refletem modos diferentes de agir.

\begin{tabular}{|c|c|c|c|c|c|}
\hline 1. Acredito que não tenho que mudar a minha voz. & DT & $\mathrm{D}$ & NS & $\mathrm{C}$ & CT \\
\hline 2. Eu acho que estou pronto para melhorar minha voz. & DT & $\mathrm{D}$ & NS & $\mathrm{C}$ & CT \\
\hline 3. O meu problema de voz me incomoda e eu estou tentando resolvê-lo & DT & $\mathrm{D}$ & NS & C & $\mathrm{CT}$ \\
\hline 4. Acho que vale a pena cuidar da minha voz. & DT & $\mathrm{D}$ & NS & $\mathrm{C}$ & CT \\
\hline 5. Eu não tenho um problema na voz. Não faz sentido para mim, mudá-la. & DT & $\mathrm{D}$ & NS & $\mathrm{C}$ & CT \\
\hline 6. Fico preocupado em ter um novo problema de voz, por isto estou procurando ajuda. & DT & $\mathrm{D}$ & NS & $\mathrm{C}$ & CT \\
\hline 7. Finalmente estou tratando o meu problema de voz. & DT & $\mathrm{D}$ & NS & $\mathrm{C}$ & CT \\
\hline 8. Eu acho que quero mudar a minha voz. & DT & $\mathrm{D}$ & NS & $\mathrm{C}$ & CT \\
\hline 9. Tenho tido sucesso no meu tratamento de voz, mas não tenho certeza se consigo mantê-la boa sem ajuda. & DT & $\mathrm{D}$ & NS & $\mathrm{C}$ & CT \\
\hline 10. Às vezes é difícil tratar a minha voz, mas estou me dedicando para isto. & DT & $\mathrm{D}$ & NS & $\mathrm{C}$ & CT \\
\hline 11. O tratamento de voz é um desperdício de tempo para mim, pois minha voz não me incomoda. & DT & $\mathrm{D}$ & NS & C & CT \\
\hline 12. Eu espero compreender melhor o meu problema de voz. & DT & $\mathrm{D}$ & NS & $\mathrm{C}$ & CT \\
\hline 13. Eu sei que tenho um problema de voz, mas não preciso fazer nada para melhorar. & DT & $\mathrm{D}$ & NS & $\mathrm{C}$ & CT \\
\hline 14. Estou me dedicando bastante para melhorar o meu problema de voz. & DT & $\mathrm{D}$ & NS & $\mathrm{C}$ & CT \\
\hline 15. Eu tenho um problema de voz e estou certo de que vou resolvê-lo. & DT & $\mathrm{D}$ & NS & $\mathrm{C}$ & CT \\
\hline 16. Não estou conseguindo manter minha voz "boa" e quero evitar um novo problema. & DT & $\mathrm{D}$ & NS & $\mathrm{C}$ & CT \\
\hline 17. Ainda que minha voz não esteja boa o tempo todo, estou me dedicando para melhorá-la. & DT & $\mathrm{D}$ & NS & $\mathrm{C}$ & CT \\
\hline 18. Achei que depois de tratar a voz eu me livraria deste problema, mas algumas vezes ele ainda me incomoda. & DT & $\mathrm{D}$ & NS & $\mathrm{C}$ & CT \\
\hline 19. Eu gostaria de saber mais como melhorar minha voz. & DT & $\mathrm{D}$ & NS & C & CT \\
\hline 20. Eu comecei a tratar a minha voz, mas preciso de mais ajuda. & DT & $\mathrm{D}$ & NS & $\mathrm{C}$ & CT \\
\hline 21. Talvez um fonoaudiólogo ou algum tratamento possa ajudar a resolver meu problema de voz. & DT & $\mathrm{D}$ & NS & $\mathrm{C}$ & CT \\
\hline 22. Eu preciso de um incentivo para manter o que consegui mudar na minha voz. & DT & $\mathrm{D}$ & NS & $\mathrm{C}$ & CT \\
\hline 23. Talvez eu seja responsável por parte do meu problema de voz, mas não sou o único responsável. & DT & $\mathrm{D}$ & NS & $\mathrm{C}$ & CT \\
\hline 24. Eu tenho esperança que alguém me ajude a melhorar a minha voz. & DT & $\mathrm{D}$ & NS & $\mathrm{C}$ & CT \\
\hline 25. Eu já estou fazendo a minha parte para melhorar minha voz & DT & $\mathrm{D}$ & NS & $\mathrm{C}$ & CT \\
\hline $\begin{array}{l}\text { 26. Toda essa conversa sobre voz é uma chatice. Por que as pessoas não podem simplesmente esquecer } \\
\text { o problema e conviver com a voz que têm? }\end{array}$ & DT & $\mathrm{D}$ & NS & $\mathrm{C}$ & CT \\
\hline
\end{tabular}




\begin{tabular}{|c|c|c|c|c|c|}
\hline 27. Estou me esforçando muito para não ter uma recaída no meu problema de voz. & DT & $\mathrm{D}$ & NS & C & CT \\
\hline 28. É frustrante, mas eu sinto que minha voz está piorando de novo. & DT & $\mathrm{D}$ & NS & C & CT \\
\hline 29. Eu me preocupo com minha voz como todo mundo. Por que perder tempo pensando nisso? & DT & $\mathrm{D}$ & NS & $\mathrm{C}$ & CT \\
\hline 30. Eu estou trabalhando ativamente para resolver meu problema de voz. & DT & $\mathrm{D}$ & NS & C & CT \\
\hline 31. Eu prefiro lidar com meu problema de voz do que fazer um tratamento. & DT & $\mathrm{D}$ & NS & $\mathrm{C}$ & CT \\
\hline 32. Depois de tudo o que fiz para melhorar a minha voz, ela às vezes, ainda me preocupa. & DT & $\mathrm{D}$ & NS & C & CT \\
\hline
\end{tabular}

Legenda: $\mathrm{DT}$ = discordo totalmente; $\mathrm{D}=$ discordo; $\mathrm{NS}=$ não sei; $\mathrm{C}=$ concordo; $\mathrm{CT}$ = concordo totalmente

* Tradução e adaptação: Teixeira L, Gama AC, Vilar A, Girundi A, Azevedo R, Behlau M, 2010 